\title{
Cancer cell-derived exosomal circUHRF1 induces natural killer cell exhaustion and may cause resistance to anti-PD1 therapy in hepatocellular carcinoma
}

Peng-Fei Zhang ${ }^{1,2,3 \dagger}$, Chao Gao ${ }^{1,2+}$, Xiao-Yong Huang ${ }^{1,2+}$, Jia-Cheng Lu ${ }^{1,2+}$, Xiao-Jun Guo ${ }^{1,2}$, Guo-Ming Shi ${ }^{1,2^{*}}$, Jia-Bin Cai ${ }^{1,2^{*}}$ and Ai-Wu Ke $\mathrm{e}^{1,2^{*}}$ (D)

\begin{abstract}
Objective: Natural killer (NK) cells play a critical role in the innate antitumor immune response. Recently, NK cell dysfunction has been verified in various malignant tumors, including hepatocellular carcinoma (HCC). However, the molecular biological mechanisms of NK cell dysfunction in human HCC are still obscure.

Methods: The expression of circular ubiquitin-like with PHD and ring finger domain 1 RNA (circUHRF1) in HCC tissues, exosomes, and cell lines was detected by qRT-PCR. Exosomes were isolated from the culture medium of HCC cells and plasma of HCC patients using an ultracentrifugation method and the ExoQuick Exosome Precipitation Solution kit and then characterized by transmission electronic microscopy, NanoSight and western blotting. The role of circUHRF1 in NK cell dysfunction was assessed by ELISA. In vivo circRNA precipitation, RNA immunoprecipitation, and luciferase reporter assays were performed to explore the molecular mechanisms of circUHRF1 in NK cells. In a retrospective study, the clinical characteristics and prognostic significance of circUHRF1 were determined in HCC tissues.

Results: Here, we report that the expression of circUHRF1 is higher in human HCC tissues than in matched adjacent nontumor tissues. Increased levels of circUHRF1 indicate poor clinical prognosis and NK cell dysfunction in patients with HCC. In HCC patient plasma, circUHRF1 is predominantly secreted by HCC cells in an exosomal manner, and circUHRF1 inhibits NK cell-derived IFN- $\gamma$ and TNF-a secretion. A high level of plasma exosomal circUHRF1 is associated with a decreased NK cell proportion and decreased NK cell tumor infiltration. Moreover, circUHRF1 inhibits NK cell function by upregulating the expression of TIM-3 via degradation of miR-449c-5p. Finally, we show that circUHRF1 may drive resistance to anti-PD1 immunotherapy in HCC patients.
\end{abstract}

Conclusions: Exosomal circUHRF1 is predominantly secreted by HCC cells and contributes to immunosuppression by inducing NK cell dysfunction in HCC. CircUHRF1 may drive resistance to anti-PD1 immunotherapy, providing a potential therapeutic strategy for patients with HCC.

\footnotetext{
* Correspondence: shi.guoming@zs-hospital.sh.cn; cai.jiabin@zs-hospital.sh.cn; keaiwu_fd@hotmail.com

'Peng-Fei Zhang, Chao Gao, Xiao-Yong Huang and Jia-Cheng Lu contributed equally to this work.

'Liver Cancer Institute, Zhongshan Hospital of Fudan University, 180 Fenglin Road, Shanghai, People's Republic of China 200032

Full list of author information is available at the end of the article
}

(c) The Author(s). 2020 Open Access This article is licensed under a Creative Commons Attribution 4.0 International License, which permits use, sharing, adaptation, distribution and reproduction in any medium or format, as long as you give appropriate credit to the original author(s) and the source, provide a link to the Creative Commons licence, and indicate if changes were made. The images or other third party material in this article are included in the article's Creative Commons licence, unless indicated otherwise in a credit line to the material. If material is not included in the article's Creative Commons licence and your intended use is not permitted by statutory regulation or exceeds the permitted use, you will need to obtain permission directly from the copyright holder. To view a copy of this licence, visit http://creativecommons.org/licenses/by/4.0/ The Creative Commons Public Domain Dedication waiver (http://creativecommons.org/publicdomain/zero/1.0/) applies to the data made available in this article, unless otherwise stated in a credit line to the data. 


\section{Introduction}

Hepatocellular carcinoma (HCC) is the fifth most common cancer and the second leading cause of cancer death in the world [1]. However, despite the rapid advancements in diagnosis, surgical techniques, targeted therapy, and immunotherapy, the 5-year overall survival rate of HCC patients remains unsatisfactory due to relapse with distant metastasis and resistance to antitumor agents [2-4]. The underlying biological molecular mechanisms of $\mathrm{HCC}$ tumorigenesis, metastasis, and resistance to anti-HCC agents remain obscure [5-7]. Therefore, further exploration of HCC tumorigenesis and progression mechanisms will provide new promising therapeutic strategies for HCC.

$\mathrm{T}$ cell immunoglobulin and mucin domain 3 (TIM-3) is an immunomodulatory receptor that engages with ligands on tumor cells and the microenvironment to inhibit antitumoral immunity in a variety of cancers, including HCC [8-10]. TIM-3 is one of the major inhibitory receptors on natural killer (NK) cells, and NK cells with forced TIM-3 expression have a reduced ability to mediate antitumoral immunity [11]. Furthermore, blockade of TIM-3 may represent a novel strategy to increase NK function in cancer patients [11]. In addition, a higher density of tumoral NK cells is associated with a response to anti-PD1 therapy in tumors $[12,13]$. Importantly, a previous study reported that increased TIM-3 expression was detected in NK-92 cells transfected with an HBV expression vector and NK cells isolated from the livers of HBV transgenic mice [10]. Moreover, blockade of TIM-3 resulted in increased cytotoxicity of NK cells against HCC cells, as well as increased interferongamma (IFN- $\gamma$ ) production [10]. However, research on NK cells in HCC has been relatively scarce despite considerable evidence showing that they have an important role in malignancy.

Ubiquitin-like with PHD and RING finger domain 1 (UHRF1) is a critical molecule that participates in regulating DNA methylation and is usually overexpressed in many cancers, including HCC [14]. Importantly, forced UHRF1 expression promotes HCC tumorigenesis and progression [14]. Therefore, we speculated that UHRF1derived circRNA expression might be upregulated and might promote the progression of HCC. Here, we analyzed UHRF1-derived circRNA expression profiles in human HCC tissues, adjacent nontumor tissues, and HCCderived exosomes and identified circUHRF1 (hsa_circ 0048677) as a significantly increased circRNA in HCC tissues. Furthermore, the expression of circUHRF1 was closely related to poor prognosis in HCC patients. Additionally, we found that HCC-derived exosomal circUHRF1 upregulates the expression of the miR-449c-5p target gene TIM-3 in NK cells by degrading miR-449c$5 \mathrm{p}$, thereby promoting immune evasion and resistance to anti-PD1 immunotherapy in HCC. Thus, circUHRF1 might act as a promising therapeutic target in $\mathrm{HCC}$ patients.

\section{Methods}

Cell lines and clinical tissues

Six human HCC cell lines (HepG2, HCCLM3, SMMC7721, Huh 7, PLC/PRF/5, and Hep3B) were cultured in Dulbecco's modified Eagle's medium (DMEM, HyClone, Cat: SH30243) supplemented with 10\% fetal bovine serum (FBS, Gibco, Cat: 10100147). The NK-92 cell line was cultured in RPMI-1640 (HyClone, Cat: SH30809) supplemented with 20\% FBS and $150 \mathrm{IU} / \mathrm{mL}$ recombinant human interleukin-2 (IL-2) (Novoprotein, Shanghai, Cat: GMP-C013). The K562 cell line was cultured in RPMI-1640 supplemented with $10 \%$ FBS. All of the above cell lines were cultured at $37^{\circ} \mathrm{C}$ in a $5 \% \mathrm{CO}_{2}$ incubator.

The tissue samples used in this study were collected as described in Additional file 1: Supplementary Materials and Methods.

\section{Exosome isolation and electron microscopy}

Exosomes from the serum of HCC patients and culture medium of HCC cells were isolated using ExoQuick Exosome Precipitation Solution (SBI System Biosciences, Cat: EXOQ5A-1) according to the manufacturer's instructions. Then, the exosomes were examined by transmission electron microscopy as described previously [15-17].

\section{Quantitative real-time polymerase chain reaction (qRT- PCR) analysis and western blotting analysis}

qRT-PCR and western blotting analyses were performed as described previously and in Additional file 1: Supplementary Materials and Methods [18]. The primers and antibodies used in this study are listed in Additional file 2: Supplementary Tables 1 and 2.

\section{Immunohistochemistry}

Immunohistochemistry was performed, and the intensity of the positive staining was measured as described in our previous study [18]. The anti-NKG2D antibody used in this study is listed in Additional file 2: Supplementary Table 2 .

\section{RNA immunoprecipitation (RIP)}

RIP assays were performed using a Magna RIP RNA Binding Protein Immunoprecipitation Kit (Millipore, Cat: 17-770) according to the manufacturer's instructions. The anti-Argonaute 2 (AGO2) and IgG antibodies used in this study are listed in Additional file 2: Supplementary Table 2. 


\section{In vivo circRNA precipitation (circRIP) assay}

Biotin-labeled circUHRF1 and negative control probes (Supplementary Table 3) were synthesized by GenePharma (Shanghai, China). The circRIP assay was performed as described previously [18]. The sequence of the circUHRF1 probe is listed in Additional file 2: Supplementary Table 3 .

\section{Luciferase reporter assay}

The wild-type TIM-3 3' UTR and circUHRF1 sequences were cloned into a pLG3 plasmid. The mutant TIM-3 3' UTR and circUHRF1 pLG3 plasmids were generated using a mutagenesis kit (Qiagen, USA, Cat: 200521) according to the manufacturer's instructions. Healthy donor-derived NK cells and NK-92 cells were seeded into 96-well plates and cotransfected with a luciferase reporter vector and miR-449c-5p mimics or the negative control using the Lipofectamine 2000 transfection reagent (Thermo Fisher, Cat: 11668-019). After $48 \mathrm{~h}$, the firefly and Renilla luciferase activities were quantified with the Dual-Luciferase Reporter Assay System (Promega, USA, Cat: E1910).

\section{Preparation of purified NK cells and $\mathrm{CD} 8^{+} \mathrm{T}$ cells}

NK cells $\left(\mathrm{CD}^{+} 6^{+} / \mathrm{CD} 56^{+}\right)$(Miltenyi, Cat: 130-092-660) and $\mathrm{CD}^{+} \mathrm{T}$ cells (Miltenyi, Cat: 130-045-201) were obtained from peripheral blood mononuclear cells (PBMCs) of healthy donors by positive selection using magnetic cell separation according to the manufacturer's instructions.

\section{Enzyme linked immunosorbent assay (ELISA)}

The concentrations of IFN- $\gamma$ and TNF- $\alpha$ produced by NK cells and $C D 8^{+} \mathrm{T}$ cells were measured by IFN- $\gamma$ and TNF- $\alpha$ ELISA kits (eBioscience, USA, Cat: KHC4021 and Cat: BMS223HS) in accordance with the manufacturer's guidelines.

\section{RNA pulldown assay}

The pulldown assay was performed as described previously $[19,20]$. In brief, to generate probe-coated beads, the biotinylated circUHRF1 probe, biotinylated circANRIL probe, and biotinylated negative control (NC) probe (GenePharma, China) were incubated with M-280 streptavidin magnetic beads (Invitrogen, USA, Cat: 11205D) at room temperature for $2 \mathrm{~h}$. Then, approximately $1 \times$ $10^{7}$ NK-92 cells were harvested, lysed, sonicated and incubated with probe-coated beads at $4{ }^{\circ} \mathrm{C}$ overnight. Subsequently, the RNA complexes bound to the beads were eluted and extracted with an RNeasy Mini Kit (QIAGEN, USA, Cat: 74104) and analyzed by qRT-PCR assay.

\section{In vivo metastasis assays}

The in vivo metastasis assays are described in our previous studies [21] and in the Supplementary Materials and Methods.

\section{Transfection experiment}

All lentiviral vectors used in this study were purchased from Genomeditech (Shanghai, China). The circUHRF1overexpression and circUHRF1-shRNA lentiviral vectors were transfected into NK-92 cells, and the blank lentiviral vectors were used as negative controls. The circUHRF1 shRNA target sequences used in this study are listed in Additional file 2: Supplementary Table 4.

\section{In vivo anti-PD1 experiments}

Male NOD/SCID mice aged 4-6 weeks were maintained according to the stated guidelines. HCCLM3 cells $(5 \times$ $10^{6}$ ) with or without reduced circUHRF1 were injected into the mouse right flank to generate subcutaneous tumors. When, the tumor reached a volume of approximately $100 \mathrm{~mm}^{3}$, NK cells $\left(1 \times 10^{6}\right)$ were resuspended in phosphate-buffered saline and injected intravenously through the tail vein. The mice were randomly assigned to four groups. Then, the mice received tail vein injection of Opdivo or its isotype control at $100 \mu \mathrm{g}$ per dose three times a week for 2 weeks. Animals were euthanized when tumors reached a maximum of $1000 \mathrm{~mm}^{3}$ $(n=6)$. The day that the mice received the first therapy was considered day 1 . Tumor volume was calculated as (length $\mathrm{x}$ width $\left.{ }^{2}\right) / 2$.

\section{Statistical analysis}

Statistical analyses were performed with SPSS software (19.0; SPSS, Inc., Chicago, IL). Values are presented as the mean \pm standard deviation (SD). The statistical analyses are described in detail in the Supplementary Materials and Methods. $P<0.05$ was considered statistically significant.

\section{Results}

Analysis of UHRF1-related circRNAs in HCC tissues

UHRF1 is frequently upregulated and plays a critical pathological role in HCC progression [14]. Furthermore, circRNAs were found to be produced from the backsplicing of precursor mRNAs (pre-mRNAs) and are considered byproducts of pre-mRNA splicing in cancer cells [22-24]. Usually, the levels of circRNAs are in accordance with their respective mRNA levels [25]. Therefore, we examined the levels of 14 circular RNAs derived from UHRF1 by qRT-PCR (circular RNA sequencing data were obtained from the online database circBase). Among them, hsa_circ_0048677 (circUHRF1) was the most abundant circRNA and was upregulated in HCC tissues (Fig. 1a and b). Then, we examined the expression of circUHRF1 by qRT-PCR in 240 HCC tissues and matched adjacent nontumor tissues. CircUHRF1 expression in HCC tissues was significantly higher than that in adjacent nontumor tissues (tumor/adjacent nontumor tissues $\geq 2 ; 136 / 240$ ) (Fig. 1c and d). Next, we explored the relationship between circUHRF1 expression and the 


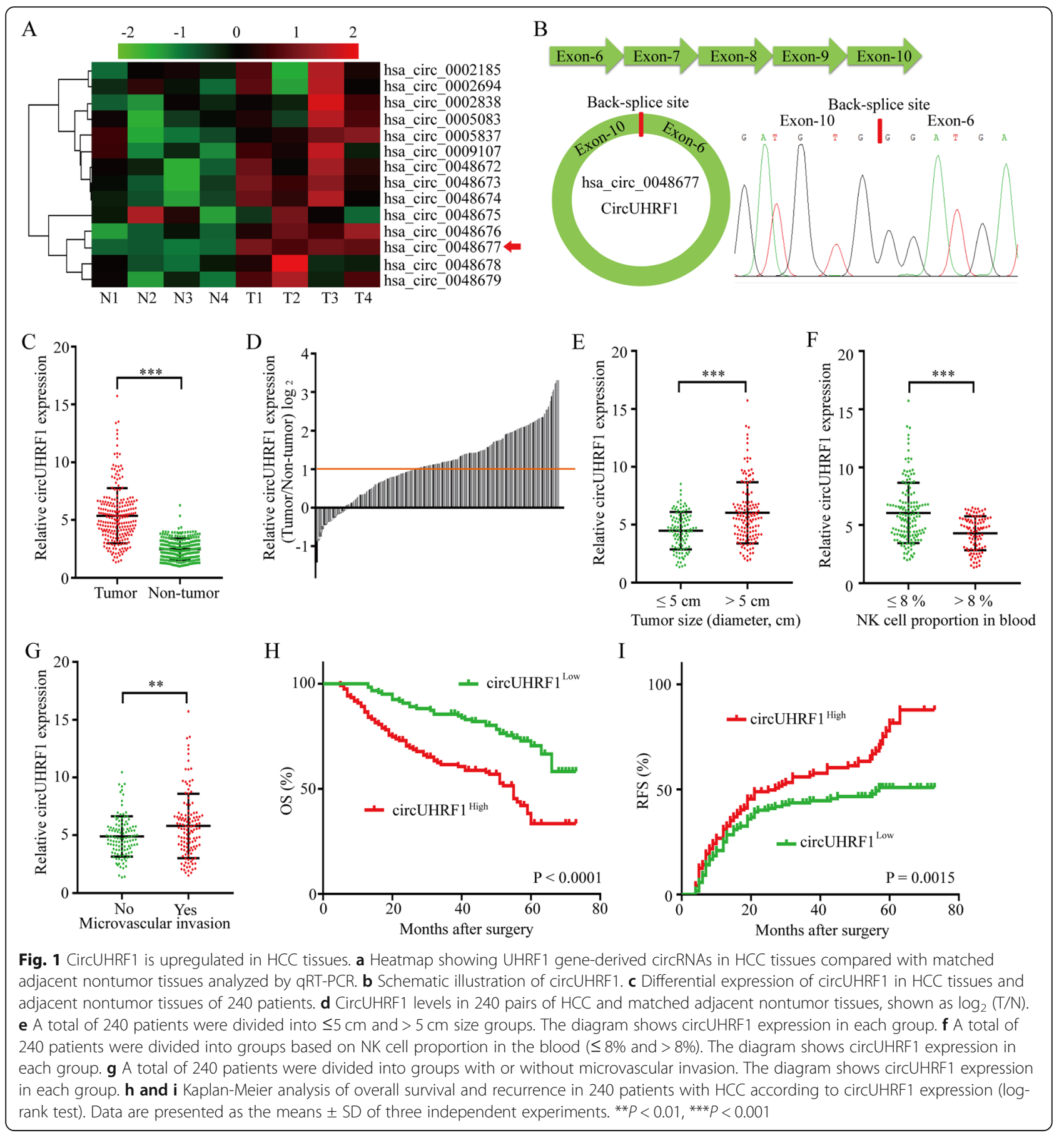

clinicopathological characteristics of 240 HCC patients, and the results are listed in Table 1. The results showed that HCC patients with circUHRF $^{\text {high }}$ expression had larger tumor size $(P=0.001)$ (Fig. 1e), lower NK cell proportions in blood $(P=0.005)$ (Fig. 1f), and more microvascular invasion $(P=0.020)$ (Fig. $1 \mathrm{~g})$ than those with circUHRF $1^{\text {low }}$ expression. Furthermore, we analyzed the prognostic potential of circUHRF1 expression in HCC patients. Importantly, Kaplan-Meier survival analysis indicated that patients with circUHRF $^{\text {high }}$ expression levels had a poor clinical prognosis (Fig. $1 \mathrm{~h}$ and i). Multivariate analysis identified circUHRF1 expression as an independent predictor of overall survival (OS) and postoperative recurrence (Tables 2 and 3). In addition, we also analyzed the clinical data in the hepatitis Bpositive patients $(216 / 240)$ and found that the findings in hepatitis B-positive patients alone were consistent with those in overall patient population, which might be 
Table 1 Correlation between circUHRF1 and clinicopathological characteristics in $240 \mathrm{HCC}$ patients

\begin{tabular}{|c|c|c|c|}
\hline \multirow[t]{2}{*}{ Variable } & \multicolumn{2}{|c|}{ circUHRF1 } & \multirow[t]{2}{*}{$P$ value } \\
\hline & Low & High & \\
\hline \multicolumn{4}{|l|}{ Age (years) } \\
\hline$\leq 50$ & 52 & 57 & \multirow[t]{2}{*}{0.604} \\
\hline$>50$ & 68 & 63 & \\
\hline \multicolumn{4}{|l|}{ Sex } \\
\hline Female & 41 & 37 & \multirow[t]{2}{*}{0.679} \\
\hline Male & 79 & 83 & \\
\hline \multicolumn{4}{|l|}{ HBsAg } \\
\hline Negative & 15 & 9 & \multirow[t]{2}{*}{0.282} \\
\hline Positive & 105 & 111 & \\
\hline \multicolumn{4}{|l|}{ HCVAb } \\
\hline Negative & 101 & 107 & \multirow[t]{2}{*}{0.343} \\
\hline Positive & 19 & 13 & \\
\hline \multicolumn{4}{|c|}{ Liver cirrhosis } \\
\hline No & 71 & 62 & \multirow[t]{2}{*}{0.299} \\
\hline Yes & 49 & 58 & \\
\hline \multicolumn{4}{|c|}{ Serum AFP, ng/mL } \\
\hline$\leq 20$ & 8 & 17 & \multirow[t]{2}{*}{0.089} \\
\hline$>20$ & 112 & 103 & \\
\hline \multicolumn{4}{|c|}{ Serum ALT, U/L } \\
\hline$\leq 75$ & 88 & 75 & \multirow[t]{2}{*}{0.097} \\
\hline$>75$ & 32 & 45 & \\
\hline \multicolumn{4}{|c|}{ Tumor size (diameter, cm) } \\
\hline$\leq 5$ & 65 & 38 & \multirow[t]{2}{*}{0.001} \\
\hline$>5$ & 55 & 82 & \\
\hline \multicolumn{4}{|c|}{ NK cell proportion in blood } \\
\hline$\leq 8 \%$ & 62 & 84 & \multirow[t]{2}{*}{0.005} \\
\hline$>8 \%$ & 58 & 36 & \\
\hline \multicolumn{4}{|c|}{ Microvascular invasion } \\
\hline No & 67 & 48 & \multirow[t]{2}{*}{0.020} \\
\hline Yes & 53 & 72 & \\
\hline \multicolumn{4}{|c|}{ Tumor number } \\
\hline Single & 84 & 76 & \multirow[t]{2}{*}{0.338} \\
\hline Multiple & 36 & 44 & \\
\hline \multicolumn{4}{|l|}{ TNM } \\
\hline$|/| \mid$ & 44 & 52 & \multirow[t]{2}{*}{0.356} \\
\hline III/IV & 76 & 68 & \\
\hline
\end{tabular}

due to the large proportion of hepatitis B-positive patients in this cohort. Taken together, our data suggest that upregulated circUHRF1 expression in HCC cells is correlated with poor outcome in patients and indicate that increased circUHRF1 expression is likely involved in the progression of $\mathrm{HCC}$.
HCC cells secrete circUHRF1 in an exosomal manner

Previous studies have reported that circRNA can be transferred from cell to cell via exosomes [26, 27]. Thus, we hypothesized that circUHRF1 could be transferred from HCC cells to other cells. To verify this hypothesis, exosomes from the supernatants of HCC cell lines were isolated and evaluated by TEM and western blotting (Fig. 2a and b). Then, qRT-PCR analyses in six HCC cell lines and paired exosomes showed that circUHRF1 was markedly upregulated in the HCCLM3 and SMMC-7721 cell lines compared with the other cell lines (Fig. 2c). Interestingly, circUHRF1 expression in exosomes was consistent with the parental cell expression levels (Fig. 2d).

Because exosomal circUHRF1 was detected in HCCderived exosomes, we explored whether circUHRF1 was present in HCC patient plasma. Importantly, circUHRF1 was present in plasma exosomal RNA. Plasma exosomal circUHRF1 levels were increased in HCC patients compared with 20 healthy donors (Fig. 2e). Furthermore, plasma exosomal circUHRF1 levels were reduced after tumor resection and increased in patients with tumor relapse, indicating that plasma exosomal circUHRF1 was mainly produced by HCC cells (Fig. 2e). Interestingly, exosomal circUHRF1 levels were significantly increased in the plasma of patients with evidence of immune evasion mechanisms, which had a decreased NK cell proportion in peripheral circulation (Fig. 2f). These data suggested that plasma exosomal circUHRF1 could serve as a critical molecular determinant of NK cell-related immune evasion.

\section{HCC cells suppress NK cell functions through exosomal circUHRF1}

To determine whether HCC-derived exosomal circUHRF1 induces alterations in allogeneic NK cells, NK cells from 6 healthy donors were cocultured with HCCLM3 and SMMC-7721 cells at a 1:1 ratio for $24 \mathrm{~h}$ (Fig. 3a). Compared with NK cells cultured alone, NK cells cocultured with SMMC-7721 and HCCLM3 cells demonstrated significant impairment of IFN- $\gamma$ and TNF$\alpha$ secretion when subsequently cocultured with $\mathrm{K} 562$ cells (Fig. 3a-c). In addition, NK cells cocultured with SMMC-7721 and HCCLM3 cells demonstrated significantly increased circUHRF1 expression (Fig. 3d), which was impaired by the exosome inhibitor GW4869 (Fig. 3e). NK cells cocultured with SMMC-7721 and HCCLM3 cells prior to treatment with an exosome inhibitor exhibited attenuated impairment of IFN- $\gamma$ and TNF- $\alpha$ secretion when subsequently cocultured with K562 cells (Fig. $3 \mathrm{f}$ and g). To further investigate whether the inhibitory effects of HCC cells on NK cell function were mediated in an exosomal circUHRF1-dependent manner, circUHRF1 knockdown cells were established using an shRNA lentiviral vector (which targeted the 
Table 2 Univariate and multivariate analyses of factors associated with overall survival

\begin{tabular}{|c|c|c|c|c|}
\hline \multirow[b]{3}{*}{ Factors } & \multicolumn{4}{|l|}{ OS } \\
\hline & \multirow[b]{2}{*}{ Univariate, $\mathrm{P}$} & \multicolumn{3}{|c|}{ Multivariate } \\
\hline & & $\mathrm{HR}$ & $95 \% \mathrm{Cl}$ & $P$ value \\
\hline Sex (Female vs. Male) & 0.771 & & & NA \\
\hline Age (years) ( $\leq 50$ vs. $>50)$ & 0.417 & & & NA \\
\hline HBsAg (Positive vs. Negative) & 0.274 & & & NA \\
\hline HCVAb (Positive vs. Negative) & 0.622 & & & NA \\
\hline Liver cirrhosis (Yes vs. No) & 0.318 & & & NA \\
\hline Serum AFP, ng/mL ( $\leq 20$ vs. $>20$ ) & 0.629 & & & NA \\
\hline Serum ALT, U/L ( $\leq 75$ vs. > 75) & 0.811 & & & NA \\
\hline Tumor size (diameter, cm) (> 5 vs $\leq 5$ ) & 0.036 & 1.622 & $1.107-2.514$ & NS \\
\hline NK cell proportion in blood ( $\leq 8 \%$ vs. > 8\%) & 0.204 & & & NA \\
\hline TNM (III/IV vs I/II.) & 0.071 & & & NA \\
\hline CircUHRF1 expression (High vs. Low) & 0.003 & 1.339 & $0.944-2.045$ & 0.022 \\
\hline
\end{tabular}

OS overall survival; NA not adopted; AFP alpha-fetoprotein; $\mathrm{HBs} A g$ hepatitis B surface antigen; $95 \% \mathrm{Cl}$ 95\% confidence interval; $H R$ hazard ratio; Cox proportional hazards regression model

back-splice site of circUHRF1) in SMMC-7721 and HCCLM3 cells (Additional file 3: Supplementary Fig. 1a). To further verify that circUHRF1 shRNA had no effects on other UHRF1 splicing products, qRT-PCR was carried out to detect UHRF1 mRNA expression. The expression of UHRF1 mRNA showed no significant change after transfection with circUHRF1 shRNA, indicating the specificity of circUHRF1 silencing (Additional file 3: Supplementary Fig. 1b). Our results also showed that exosomal circUHRF1 expression was significantly decreased in circUHRF1 knockdown cells (Fig. 3h). Interestingly, the reduction in exosomal circUHRF1 levels effectively attenuated HCC-derived exosomeinduced impairment of IFN- $\gamma$ and TNF- $\alpha$ secretion in
NK cells (Fig. $3 i$ and $j)$. These results collectively suggest that HCC-derived exosomal circUHRF1 impaired the functions of NK cells.

CircUHRF1 and miR-449c-5p degrade each other in NK cells Previous studies have reported that circRNAs mainly function as miRNA sponges to bind miRNAs and then promote miRNA-targeted gene expression. We next investigated whether circUHRF1 could bind to miRNAs in human NK-92 cells. Through StarBase v3.0 analysis, 14 miRNAs were predicted as possible targets of circUHRF1. To verify the critical miRNAs that may interact with circUHRF1 in NK-92 cells, we used a circUHRF1-specific

Table 3 Univariate and multivariate analyses of factors associated with cumulative recurrence

\begin{tabular}{|c|c|c|c|c|}
\hline \multirow[b]{3}{*}{ Factors } & \multicolumn{4}{|c|}{ Cumulative Recurrence } \\
\hline & \multirow[b]{2}{*}{ Univariate, $\mathrm{P}$} & \multicolumn{3}{|c|}{ Multivariate } \\
\hline & & $\mathrm{HR}$ & $95 \% \mathrm{Cl}$ & $P$ value \\
\hline Sex (Female vs. Male) & 0.527 & & & NA \\
\hline Age (years) ( $\leq 50$ vs. $>50)$ & 0.393 & & & NA \\
\hline HBsAg (Positive vs. Negative) & 0.266 & & & NA \\
\hline HCVAb (Positive vs. Negative) & 0.494 & & & NA \\
\hline Liver cirrhosis (Yes vs. No) & 0.802 & & & NA \\
\hline Serum AFP, ng/mL ( $\leq 20$ vs. $>20$ ) & 0.472 & & & NA \\
\hline Serum ALT, U/L ( $\leq 75$ vs. $>75$ ) & 0.615 & & & NA \\
\hline Tumor size (diameter, $\mathrm{cm}$ ) (> 5 vs $\leq 5$ ) & 0.066 & & & NA \\
\hline NK cell proportion in blood ( $\leq 8 \%$ vs. > 8\%) & 0.149 & & & NA \\
\hline TNM (III/IV vs I/II.) & 0.277 & & & NA \\
\hline CircUHRF1 expression (High vs. Low) & 0.004 & 1.762 & $1.172-2.428$ & 0.019 \\
\hline
\end{tabular}

NA not adopted; AFP alpha-fetoprotein; HBsAg hepatitis B surface antigen; 95\%Cl 95\% confidence interval; BCLC Barcelona-Clinic Liver Cancer, HR hazard ratio; Cox proportional hazards regression model 


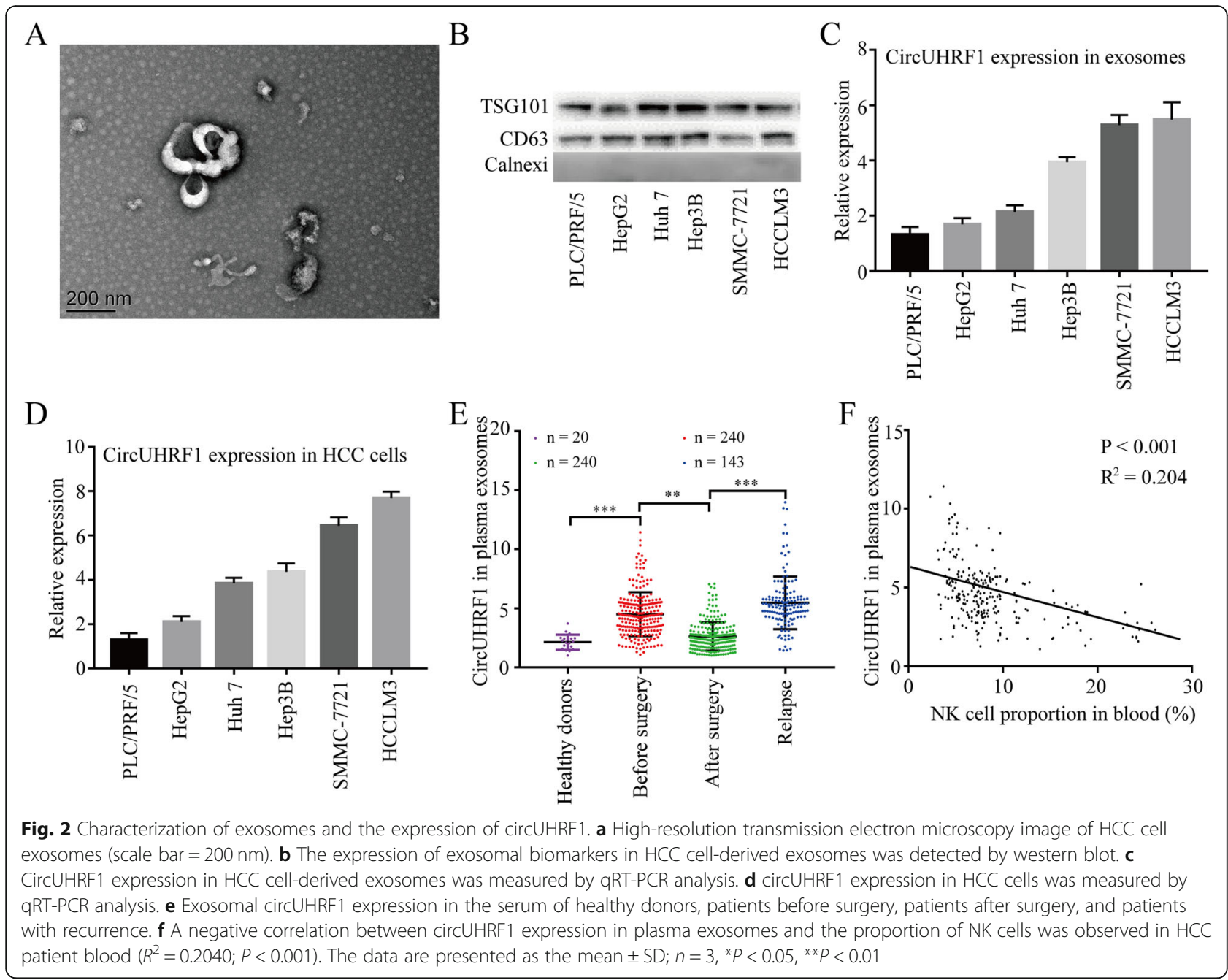

probe to carry out circRNA in vivo precipitation (circRIP) and detected the 14 predicted miRNAs in complex with circUHRF1 using qRT-PCR. Our results showed a significant enrichment of circUHRF1 and miR-449c-5p compared with the negative controls, while the other miRNAs had no enrichment or only slight enrichment (Fig. 4a), indicating that miR-449c-5p is a circUHRF1-interacting miRNA in NK-92 cells. Given that circRNAs act as miRNA sponges $[18,28,29]$, we wondered whether circUHRF1 can interact with certain miRNAs in NK cells. Next, we conducted RIP with an anti-AGO2 antibody in NK-92 cells. The results showed that circUHRF1 and miR-449c-5p, but not circANRIL (a circular RNA reported to not bind to AGO2 [30]), were significantly enriched by the anti-AGO2 antibody (Fig. 4b). This result indicated that circUHRF1 may act as a binding platform for miR-449c-5p and AGO2. To further verify this result, a biotinylated miR-449c-5p pulldown assay was performed, and the results showed significant enrichment of circUHRF1 compared with the negative control in NK-92 cells (Fig. 4c). Next, we carried out a luciferase assay using
miR-449c-5p mimics cotransfected with a pGL3 luciferase plasmid expressing the wild-type circUHRF1 sequence or an miR-449c-5p binding site-mutant circUHRF1 sequence into NK-92 cells and normal NK cells (derived from a healthy donor) (Fig. 4d). Compared with the negative control, miR-449c-5p mimics significantly reduced the luciferase reporter activity of the wild-type but not the miR449c-5p-binding site-mutant pGL3 luciferase plasmid (Fig. 4e; Additional file 3: Supplementary Fig. 2). Moreover, the level of circUHRF1 was significantly decreased after overexpression of miR-449c-5p, and the level of miR-449c-5p was significantly decreased after overexpression of circUHRF1 in NK-92 cells (Additional file 3: Supplementary Fig. 3a and b; Fig. 3f and g). These findings suggest that circUHRF1 and miR-449c-5p may target each other. All of the above results confirmed that circUHRF1 and miR-449c-5p affected each other in NK cells.

Therefore, we speculated that circUHRF1 may inhibit miR-449c-5p activity to promote functional impairment of NK cells. To test this hypothesis, we ectopically overexpressed circUHRF1 together with miR-449c-5p in NK- 


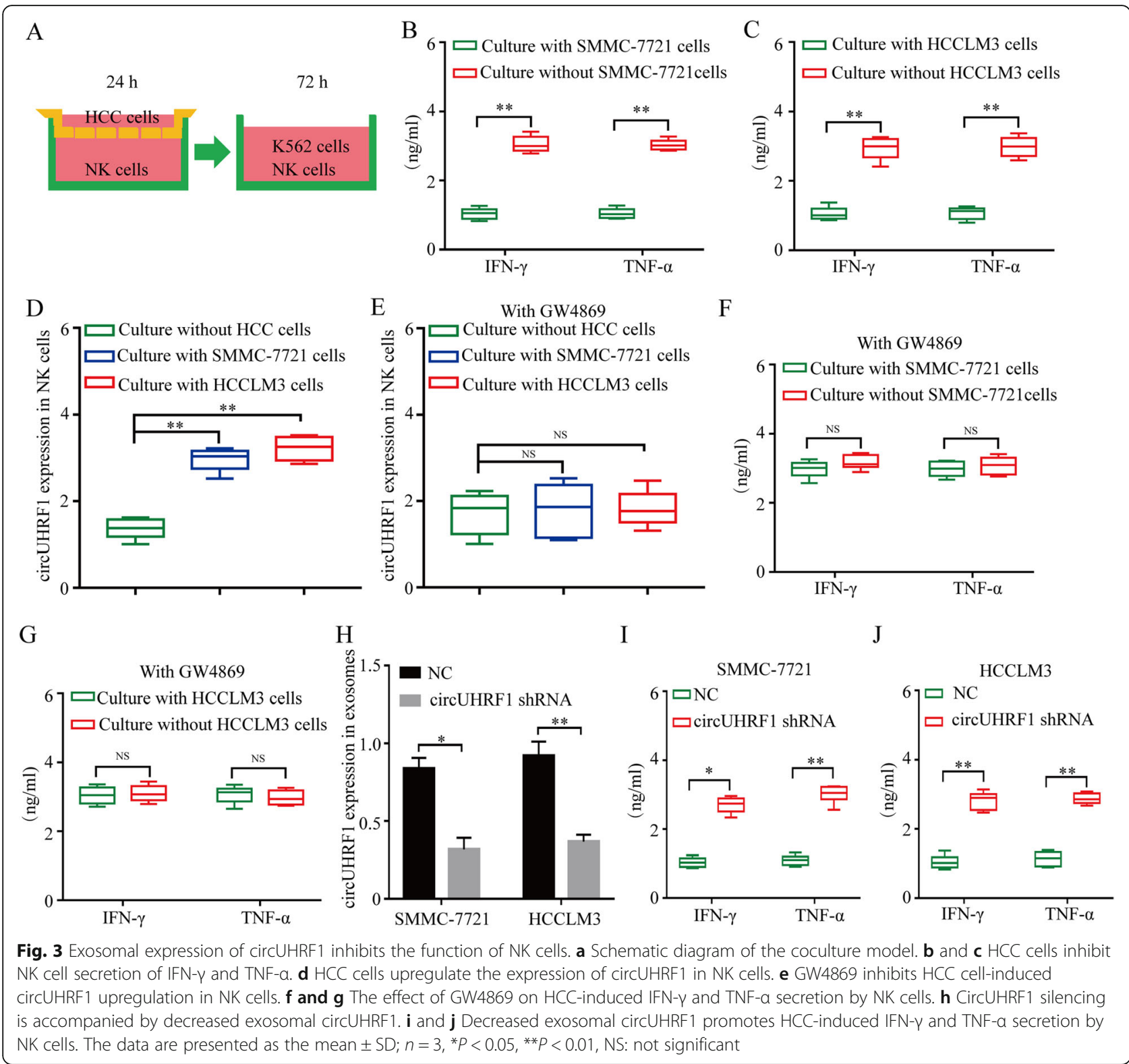

92 cells to evaluate the regulation of miR-449c-5p by circUHRF1 (Additional file 3: Supplementary Fig. 4a). Although forced miR-449c-5p expression alone showed significant tumor-suppressive activity (promoting IFN- $\gamma$ and TNF- $\alpha$ secretion), increased circUHRF1 expression dramatically inhibited the miR-449c-5p tumor-suppressive function (Additional file 3: Supplementary Fig. 4b), highlighting the critical ability of HCC-derived exosomal circUHRF1 to inhibit the function of NK cells via the miR-449c-5p-related pathway.

\section{CircUHRF1 upregulates TIM-3 expression via suppression of miR-449c-5p activity}

As our data showed that circUHRF1 effectively inhibited the function of miR-449c-5p to suppress NK cell secretion of IFN- $\gamma$ and TNF- $\alpha$, we speculated that circUHRF1 may be responsible for upregulating the expression levels of miR-449c-5p targets by acting as a miR$449 \mathrm{c}-5 \mathrm{p}$ sponge and enhancing immune evasion in $\mathrm{HCC}$ patients. To verify this hypothesis, we first analyzed a panel of predicted miR-449c-5p target genes in NK cells, which included TIM-3 (Fig. 5a). Next, we carried out a luciferase reporter assay. The pGL3 luciferase plasmid was created based on firefly luciferase expressing the wild-type 3' UTR of TIM-3 or 3' UTR TIM-3 sequence with a mutated miR-449c-5p-binding site. NK-92 cells and healthy donor NK cells were cotransfected with the pGL3 luciferase plasmid along with negative control or miR-449c-5p mimics and assayed for luciferase activity. Compared with the negative control, miR-449c-5p 
A
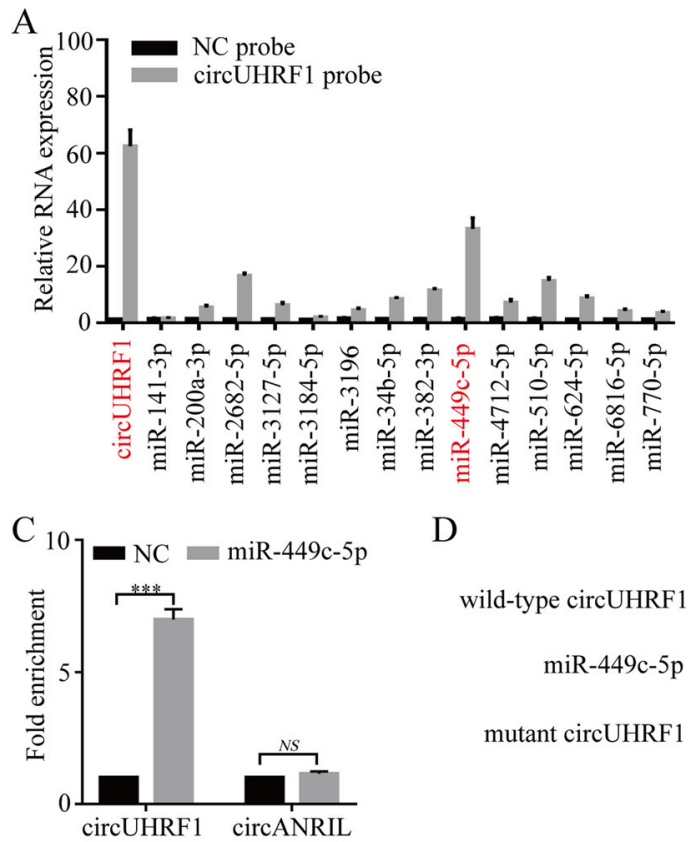

$\mathrm{E}$

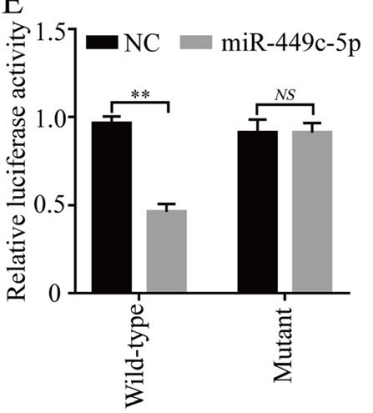

D

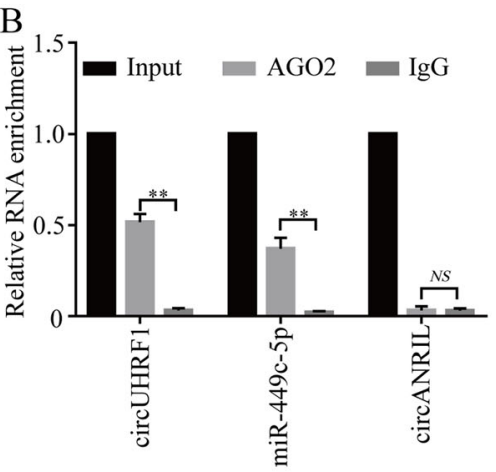

wild-type circUHRF1 5' acauggcCUUCCACAUCUACUGCCUg 3'

|| || || : || |||||

miR-449c-5p 3' ugucggcGAUCGU- UAUGUGACGGAu 5'

|| || ||: |||||||

mutant circUHRF1 5' acauggcCUUCCACAUCUUGACGGAg 3'

$\mathrm{F}$

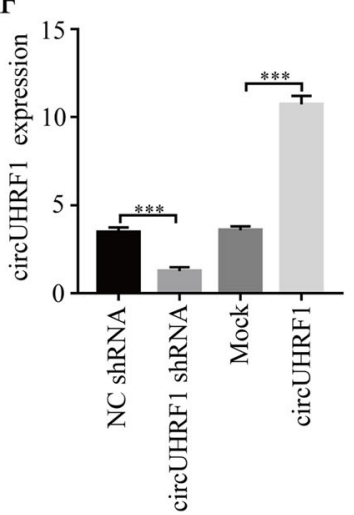

G

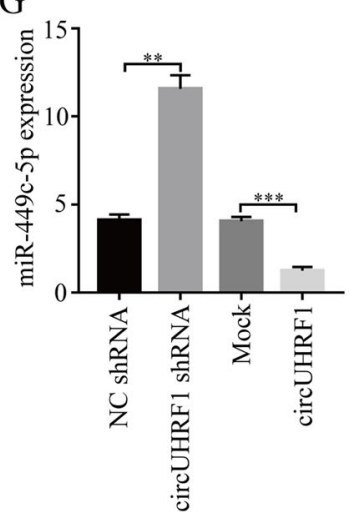

Fig. 4 CircUHRF1 inhibits the biological function of miR-449c-5p in NK cells. a RIP was performed for circRNA in NK-92 cells using a circUHRF1 probe and a negative control (NC) probe. b RIP experiments were carried out using an anti-AGO2 antibody with NK-92 cell extracts. c The level of circUHRF1 in the streptavidin-captured fractions of NK-92 cell lysates after transfection with biotinylated miR-449c-5p or the negative control (NC). CircANRIL was used as a negative control. d Putative binding sites of miR-449c-5p on circUHRF1 were predicted by StarBase v3.0. e The luciferase activity of pGL3-circUHRF1 in NK-92 cells after cotransfection with miR-449c-5p. $\mathbf{f}$ CircUHRF1 expression in NK-92 cells was modified by lentivirusmediated overexpression and knockdown. $\mathbf{g}$ The relative level of miR-449c-5p was measured by qRT-PCR in NK-92 cells transfected with circUHRF1, shcircUHRF1, mock, or the negative control. The data are presented as the mean $\pm \mathrm{SD} ; n=3,{ }^{* *} P<0.01,{ }^{* * *} P<0.001$, NS: not significant

mimics significantly reduced the luciferase reporter activity of wild-type TIM-3 (Fig. 5b). Interestingly, we verified that TIM-3 expression was significantly decreased after forced expression of miR-449c-5p (Fig. 5c and d).

In light of the critical role of TIM-3-related pathways in immune evasion, we hypothesized that circUHRF1 may be an important contributor to HCC immune evasion via its competitive circRNA activity. Our results showed that TIM-3 mRNA is a target of miR-449c-5p in NK cells. Therefore, continuous upregulation of exosomal circUHRF1 sequesters miR-449c-5p in NK cells and thereby leads to immune evasion in HCC. To further validate our hypothesis, we detected TIM-3 expression in NK-92 cells transfected with miR-449c-5p mimic alone or together with circUHRF1. The results showed that forced miR-449c-5p expression led to decreased mRNA and protein expression levels of TIM-3, while circUHRF1 reversed this inhibitory effect of miR-449c$5 \mathrm{p}$ on TIM-3 expression (Fig. 5b and c).

To further confirm our in vitro results that circUHRF1 impeded NK cell function through regulation of the miR-449c-5p/TIM-3 axis, we investigated the correlation between circUHRF1 and miR-449c-5p/TIM-3 expression in another $40 \mathrm{HCC}$ patient peripheral NK cells. We 


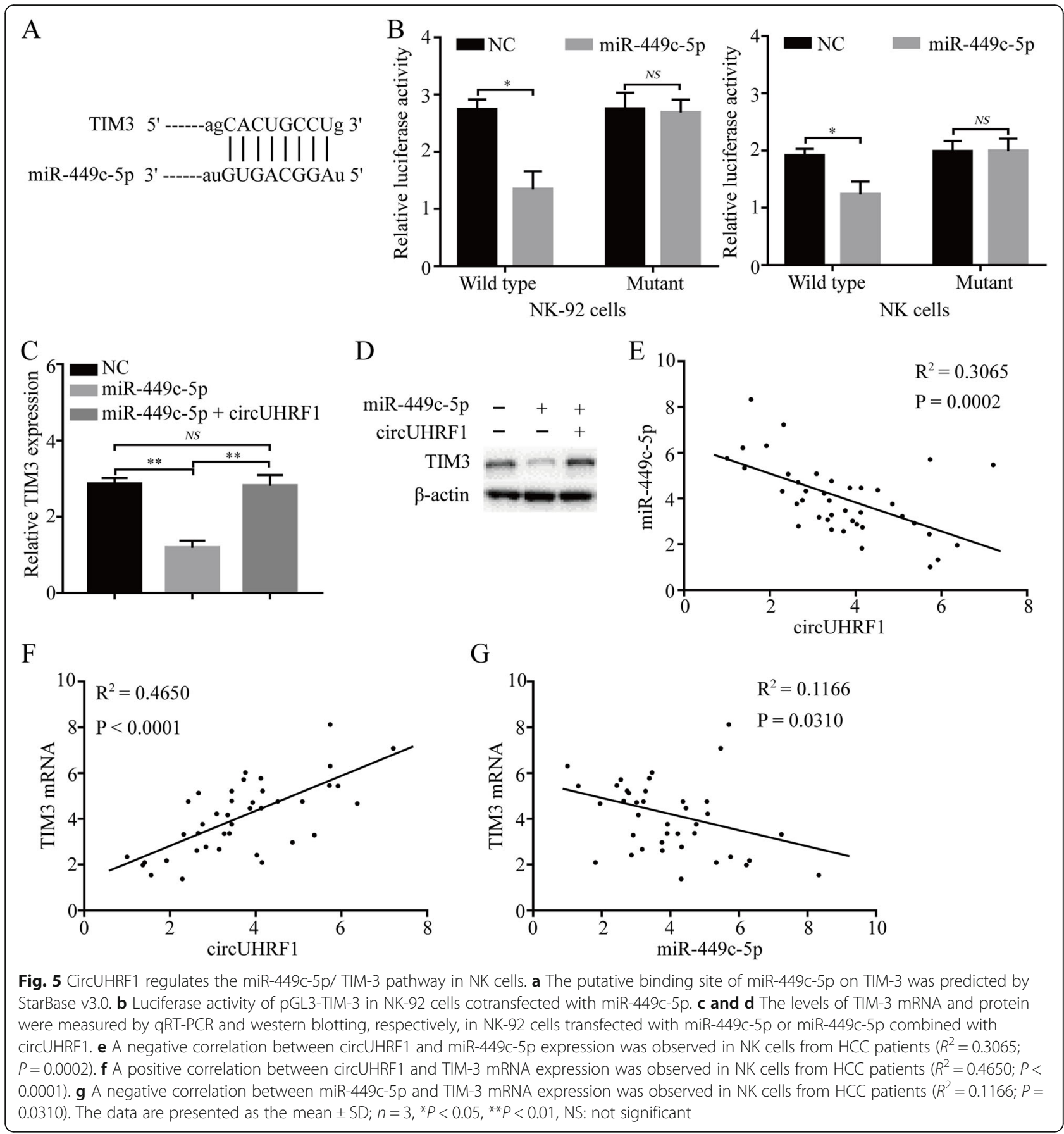

found that circUHRF1 expression was negatively correlated with miR-449c-5p expression in peripheral NK cells $\left(R^{2}=0.3065 ; P=0.0002\right.$; Fig. $\left.5 \mathrm{e}\right)$. As expected, we also found that circUHRF1 expression positively correlated with TIM-3 mRNA expression in peripheral NK cells $\left(R^{2}=0.4650 ; P<0.0001\right.$; Fig. 5f). Notably, we observed that miR-449c-5p expression was negatively associated with the expression of TIM-3 mRNA in peripheral NK cells $\left(R^{2}=0.1166 ; P=0.0310\right.$; Fig. $\left.5 \mathrm{~g}\right)$, indicating that the upregulation of exosomal circUHRF1 abrogates the tumor-suppressive effect of miR-449c-5p on TIM-3 in NK cells.

Previous studies have reported that both NK cells and $\mathrm{CD}^{+} \mathrm{T}$ cells infiltrating tumors have increased TIM-3 expression [31-33]. Therefore, we performed experiments to explore whether HCC-derived exosomal circUHRF1 inhibits the function of $\mathrm{CD}^{+} \mathrm{T}$ cells. Regrettably, compared with $\mathrm{CD}^{+} \mathrm{T}$ cells cultured alone, $\mathrm{CD}^{+} \mathrm{T}$ cells cocultured with SMMC-7721 and HCCLM3 cells demonstrated no significant impairment 
of IFN- $\gamma$ and TNF- $\alpha$ secretion when subsequently cocultured with K562 cells (Supplementary Fig. 5a and b). In addition, $\mathrm{CD}^{+} \mathrm{T}$ cells cocultured with SMMC-7721 and HCCLM3 cells demonstrated significantly increased circUHRF1 expression (Supplementary Fig. 5c). By determining miR-449c-5p expression in $\mathrm{CD} 8^{+} \mathrm{T}$ and NK cells, we found that the difference in the abundance of miR-449c-5p was obvious, and was higher in NK cells than in $\mathrm{CD}^{+}{ }^{+} \mathrm{T}$ cells (Supplementary Fig. 5d). These results might be indicate that the circUHRF $1 / \mathrm{miR}-449 \mathrm{c}-$ $5 p / T I M-3$ axis is specific to NK cells and does not function in $\mathrm{CD}^{+} \mathrm{T}$ cells.

\section{CircUHRF1 induces HCC progression in an NK cell- dependent manner}

To further explore the function of circUHRF1 in HCC, we used the pLO5-ciR-circUHRF1 lentivirus vector to upregulate the expression of circUHRF1 in PLC/PRF/5 cells. qRT-PCR results showed that the circUHRF1 levels in PLC/PRF/5 cells transfected with pLO5-ciRcircUHRF1 were significantly higher than those in the cells transfected with pLO5-ciR-Mock (Fig. 6a). Importantly, exosomes derived from HCC cells transfected with pLO5-ciR-circUHRF1 had higher levels of circUHRF1 than those derived from pLO5-ciR-Mock-transfected HCC cells (Fig. 6b). Next, we generated a NOD/SCID mouse model (injected with NK cells derived from healthy donors) of tumor lung metastasis using PLC/ PRF/5 cells. The results showed that the number of metastatic tumor nodules induced by PLC/PRF/5 cells transfected with pLO5-ciR-circUHRF1 was significantly increased compared with that induced by HCC cells transfected with pLO5-ciR-Mock (Fig. 6c). Importantly, the mice with tumor lung metastases derived from PLC/PRF/ 5 cells with forced circUHRF1 expression showed an increased circUHRF1 level in serum exosomes (Fig. 6d). Additionally, the number of NKG2D-positive cells in metastatic tumor nodules induced by HCC cells transfected with pLO5-ciR-circUHRF1 was significantly decreased compared with that in tumors induced by HCC cells transfected with pLO5-ciR-Mock (Fig. 6e). These results indicate that circUHRF1 promotes $\mathrm{HCC}$ progression in an exosome- and NK cell-dependent manner.

\section{CircUHRF1 enhances HCC resistance to anti-PD1 therapy}

Because forced circUHRF1 expression suppresses NK cell function in HCC, we next evaluated whether circUHRF1 overexpression can further impede the antitumor effect of anti-PD1 therapy (Opdivo). Therefore, we analyzed retrospective data from $30 \mathrm{HCC}$ patients with recurrent distant metastasis (including lung metastasis) receiving anti-PD1 immunotherapy who had undergone liver resection 2-36 months before immunotherapy. After six therapy cycles, the efficacy was assessed using
CT. Based on RECIST1.1 analysis, the results showed that there were 3 patients with partial response (PR), 7 patients with stable disease (SD), and 20 patients with progressive disease (PD) (Additional file 3: Supplementary Fig. 6). Then, circUHRF1 expression levels were measured, and the results indicated that the circUHRF1 expression levels in the PD group were much higher than those in the SD and PR groups (Fig. 7a). To further explore the relationship between circUHRF1 and immune evasion, we examined the expression of NKG2D in tissues from $30 \mathrm{HCC}$ patients. The number of NKG2D-positive cells in HCC patients resistant to antiPD1 therapy was significantly reduced compared with that in HCC patients susceptible to anti-PD1 therapy (Fig. 7b). A scatter plot analysis revealed a negative correlation between circUHRF1 expression and NKG2Dpositive cell number in HCC tissues $\left(R^{2}=0.1452 ; P=\right.$ 0.0377) (Fig. 7c). To further assess the effects of circUHRF1 on resistance to anti-PD1 therapy, we established a xenograft model by subcutaneous implantation of circUHRF1-knockdown HCCLM3 cells or the respective negative control cells. The results indicated that the implantation of circUHRF1 knockdown cells resulted in sensitivity to anti-PD1 treatment and an increase in the overall survival rate (Fig. $7 \mathrm{~d}$-f). Taken together, these findings indicate that forced circUHRF1 expression might impede the response of HCC to anti-PD1 treatment and that targeting circUHRF1 might be a promising and effective method to recover the sensitivity of HCC to anti-PD1 therapy.

\section{Discussion}

In recent years, numerous studies have verified the abnormal expression of circRNAs in a series of cancers and have revealed that many circRNAs play vital roles in modulating tumor immunosuppression, proliferation, migration, invasion, metastasis, and chemotherapy resistance $[24,34,35]$. However, the biological molecular mechanisms by which circRNAs participate in cancer remain unclear [6, 36]. Here, we report a UHRF1-derived circular RNA and evaluated its biological functions in promoting HCC immunosuppression. We found that circUHRF1 was highly expressed in HCC tissues and HCC-derived exosomes. Furthermore, we demonstrated that HCC-secreted exosomal circUHRF1 was delivered into NK cells, upregulated TIM-3 expression by sponging miR-449c-5p and in turn induced NK cell exhaustion. More importantly, we found that circUHRF1overexpressing HCC cells were characterized by resistance to anti-PD1 treatment in nude mice adoptively transferred with NK cells. This result was also proven by a retrospective study that analyzed a cohort of $30 \mathrm{HCC}$ patients treated with anti-PD1 agents. All of the above results demonstrated that exosomal circUHRF1 secreted 


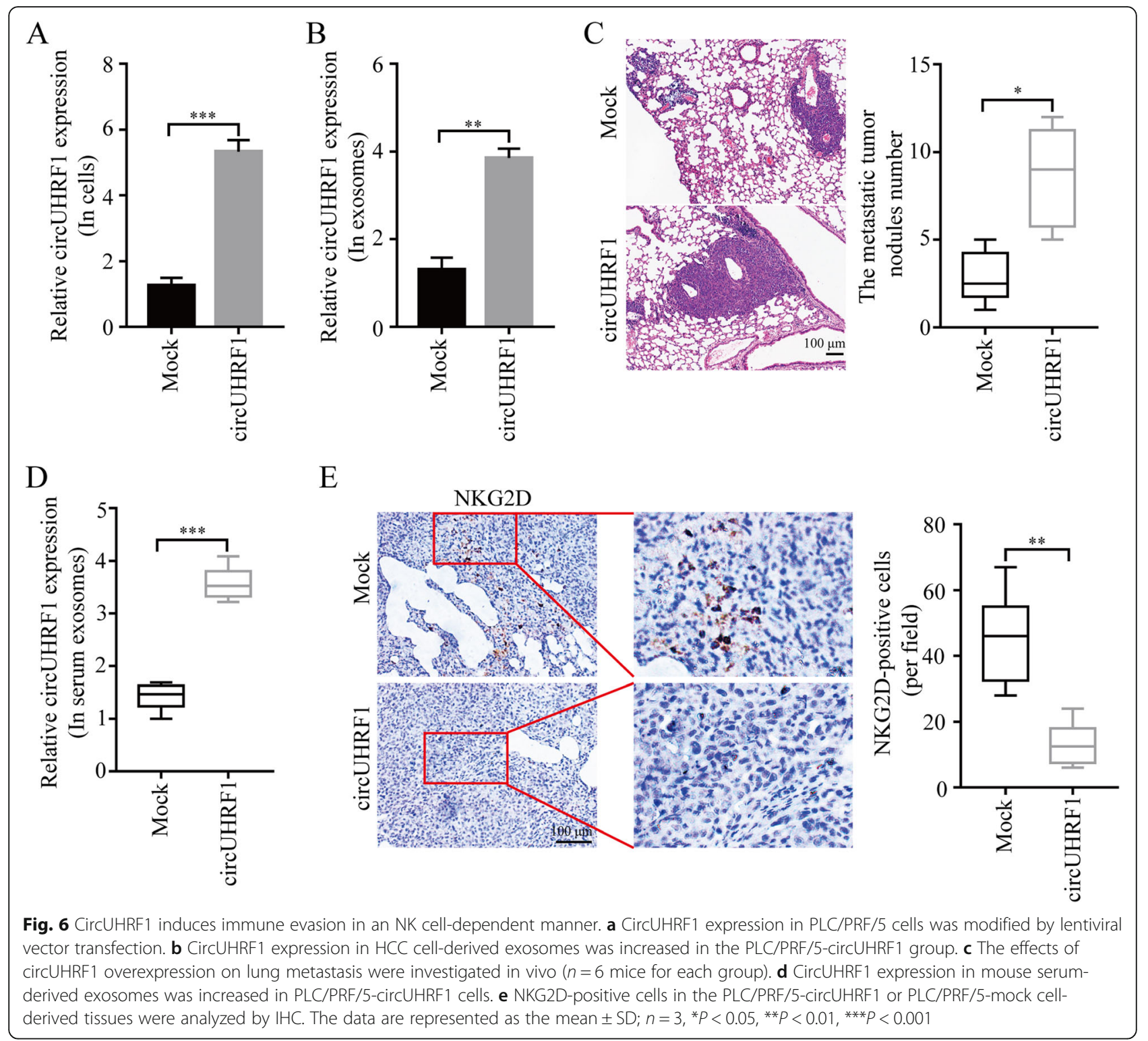

by $\mathrm{HCC}$ cells mediates resistance to anti-PD1 therapy by inducing NK cell exhaustion, which may provide a potential therapeutic strategy for patients with HCC.

Increasingly, studies have reported that intercellular communication plays a critical role in promoting the immunosuppression, proliferation, migration, and invasion of cancers [37-40]. Direct interactions, secreted biologically active molecules and exosomes are the major mechanisms for cell signal exchange [39, 40]. Usually, exosomes reflect the malignant features of donor cells and transport oncogenic signals to recipient cells that can promote cancer progression. Here, our results highlight a critical role for exosomal circUHRF1 in inducing immune evasion and resistance to anti-PD1 therapy by inducing NK cell dysfunction in HCC.
Recently, an increasing number of studies have verified exosomal circRNAs in the peripheral blood of patients with a variety of cancers, including HCC [39, 41, 42]. Importantly, circRNAs have been confirmed to participate in the regulation of various immune responses, including cancer immune evasion [34]. Our results on immune evasion and the tumor progression-promoting effects of exosomal circUHRF1 are likely to be relevant in HCC. Identifying patients with higher levels of exosomal circUHRF1 will be critical in predicting those who are more likely to be resistant to anti-PD1 immunotherapy.

NK cells have been verified to have cytotoxic effects against tumor cells in several cancers, including HCC [43-45]. PD1 is an inhibitory receptor expressed on activated lymphocytes, including $\mathrm{T}$ cells, NK cells, and $\mathrm{B}$ 


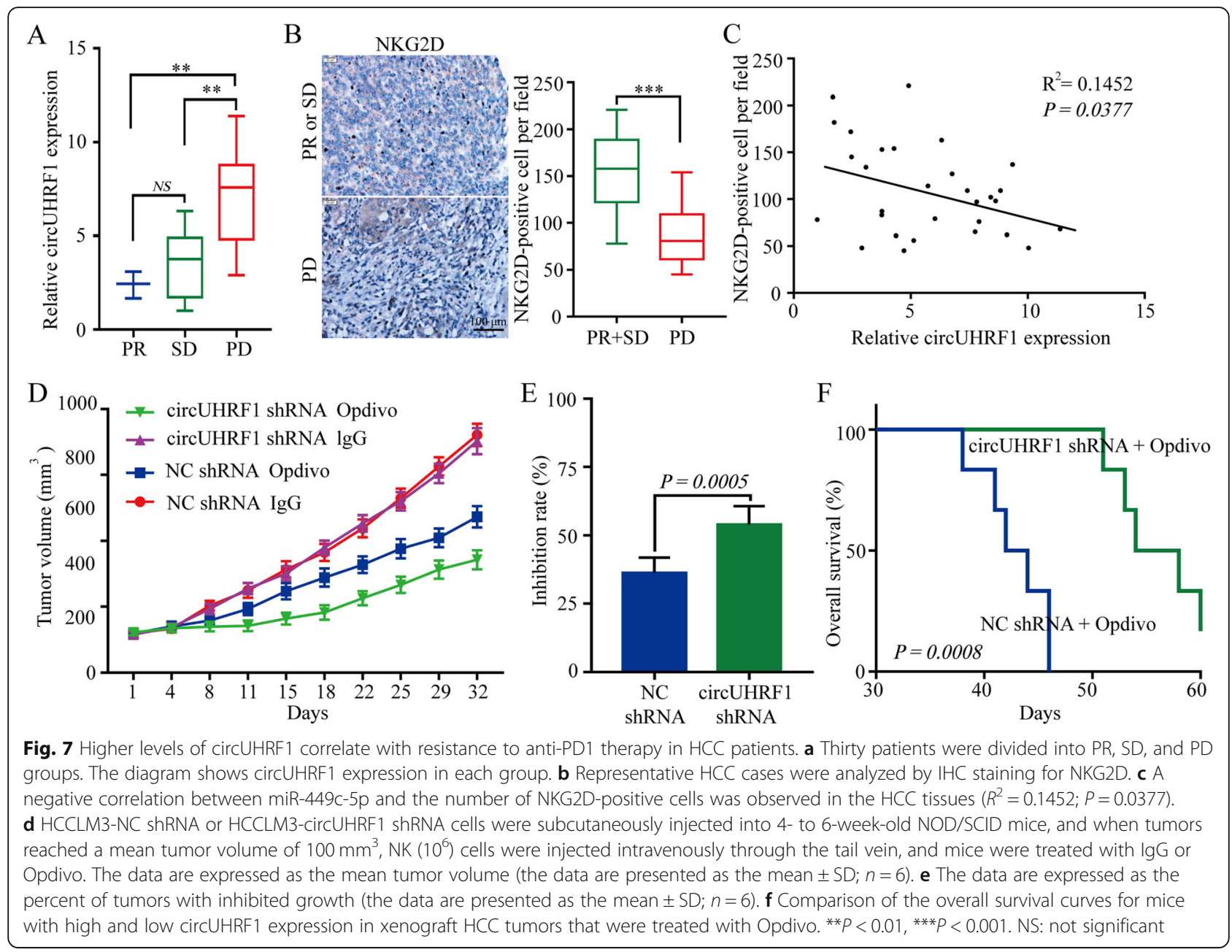

cells [46]. PD1 can be overexpressed on NK cells from HCC patients and promotes functional dysregulation of activated NK cells [47, 48]. Current research has reported that decreased NK cell infiltration in tumor tissue predicts resistance to anti-PD1 immunotherapy $[49,50]$. TIM-3 is an important coinhibitory molecule expressed on T cells and NK cells. Compared with NK cells from healthy donors, peripheral NK cells from cancer patients express significantly higher levels of surface TIM-3 [31]. Importantly, increased TIM-3 expression is associated with functional impairment and death of NK cells [31]. Previous studies have also reported that TIM-3 expression on NK cells from cancer patients transmits negative signals of NK cell cytotoxicity [32, 51]. In this study, our results showed that the expression of circUHRF1 was significantly increased not only in HCC tissues but also in HCC-derived exosomes. Furthermore, we found that higher expression of circUHRF1 was dramatically associated with poor prognosis and pathological characteristics, suggesting its tumor-promoting effect on HCC. Functionally, we demonstrated that HCC-derived exosomes suppress the ability of NK cells to produce IFN- $\gamma$ and TNF- $\alpha$ via upregulation of TIM-3 expression. In circUHRF1-overexpressing HCC cells, increased intercellular communication between NK cells and HCC cells is likely to bypass the upregulation of TIM-3, resulting in the impaired function and an exhausted phenotype of NK cells.

The unique ability of NK cells to target cancer cells without antigen specificity makes them an ideal candidate for use against those tumors [52]. Currently, the TIM-3/Gal-9 pathway has been well demonstrated in NK cells, and several studies have detected PD1 expression on NK cells in various clinical settings; thus, PD1/ PD-L1 and TIM-3/Gal-9 blockade might favor NK cell activity in antitumor immunity [53]. In this study, we showed that HCC-derived exosomal circUHRF1 can upregulate TIM-3 expression, which further induces the NK cell exhaustion. Thus we revealed that circUHRF1 levels are an important factor influencing resistance to anti-PD1 therapy in a subgroup of HCC patients.

Both NK cells and $\mathrm{CD}^{+} \mathrm{T}$ cells infiltrating tumors had increased TIM-3 expression, which indicated that anti-TIM-3 agents, as a monotherapy, might be a 
promising method for reversing the exhaustion of both NK cells and $\mathrm{CD}^{+} \mathrm{T}$ cells [31-33]. Here, we found that reduced circUHRF1 expression increased the therapeutic efficacy of anti-PD1 treatment via the exosomal circUHRF1/miR-449c-5p/TIM-3 pathway. TIM-3 expression on tumor-infiltrating NK Cells and $\mathrm{CD} 8^{+} \mathrm{T}$ cells exerts immunosuppressive effects. Anti-TIM-3 agents, as a monotherapy, might lead to benefits to patients outcomes by reversing the exhaustion of both NK cells and $\mathrm{CD} 8^{+} \mathrm{T}$ cells.

\section{Conclusions}

Overall, we have demonstrated in this study that HCCderived exosomes suppress the production of IFN- $\gamma$ and TNF- $\alpha$ via the induction of TIM-3 expression on NK cells. We also report a new mechanism by which tumor exosomes inhibit NK cell function via exosomal circUHRF1, contributing new evidence for the crosstalk between HCC cells and NK cells.

\section{Supplementary information}

Supplementary information accompanies this paper at https://doi.org/10. 1186/s12943-020-01222-5.

\section{Additional file 1. \\ Additional file 2 . \\ Additional file 3.}

\section{Abbreviations}

HCC: Hepatocellular carcinoma; OS: Overall survival; RFS: Recurrence-free survival; NKG2D: Natural-killer group 2 member D; TIM-3: T cell immunoglobulin and mucin domain 3; NK cell: Natural killer cell; qRTPCR: Quantitative real-time polymerase chain; RIP: RNA immunoprecipitation; AGO2: Argonaute 2; UHRF1: Ubiquitin like with PHD and ring finger domains 1; PD1: Programmed cell death 1; CircRNA: Circular RNA; CCK-8: Cell Counting Kit-8; IHC: Immunohistochemistry; TMA: Tissue microarray

\section{Acknowledgements}

Not applicable.

\section{Declarations}

The authors declare that they have no competing interests.

\section{Authors' contributions}

Jia-Bin Cai, Guo-Ming Shi, and Ai-Wu Ke conceived and designed the experiments; Peng-Fei Zhang, Chao Gao, Xiao-Yong Huang, and Jia-Cheng Lu performed the experiments; Peng-Fei Zhang and Xiao-Jun Guo analyzed the data; Jia-Bin Cai, Peng-Fei Zhang and Ai-Wu Ke wrote the paper. All authors read and approved the final manuscript.

\section{Funding}

This study was funded by the National Natural Science Foundation of China (81672825, 81972232, and 81702868).

\section{Availability of data and materials}

All data generated or analyzed during this study are included either in this article or in the supplementary information files.

\section{Ethics approval and consent to participate}

Ethical approval was obtained from the Zhongshan Hospital Research Ethics Committee (NO. Y2016-025), and written informed consent was obtained from each patient.
Consent for publication

Not applicable.

\section{Competing interests}

The authors declare that they have no competing interests.

\section{Author details}

${ }^{1}$ Liver Cancer Institute, Zhongshan Hospital of Fudan University, 180 Fenglin Road, Shanghai, People's Republic of China 200032. ${ }^{2}$ Key Laboratory of Carcinogenesis and Cancer Invasion, Ministry of Education, Shanghai 200032, People's Republic of China. ${ }^{3}$ Department of Medical Oncology, Zhongshan Hospital, Fudan University, Shanghai 200032, P.R. China.

Received: 13 December 2019 Accepted: 28 May 2020

Published online: 27 June 2020

\section{References}

1. Heimbach JK, Kulik LM, Finn RS, Sirlin CB, Abecassis MM, Roberts LR, Zhu AX, Murad MH, Marrero JA. AASLD guidelines for the treatment of hepatocellular carcinoma. Hepatology. 2018;67(1):358-80

2. Stotz M, Gerger A, Haybaeck J, Kiesslich T, Bullock MD, Pichler M. Molecular targeted therapies in hepatocellular carcinoma: past, Present and Future. Anticancer Res. 2015:35(11):5737-44.

3. Greten TF, Lai CW, Li G, Staveley-O'Carroll KF. Targeted and immune-based therapies for hepatocellular carcinoma. Gastroenterology. 2019;156(2):51024.

4. Lin DC, Mayakonda A, Dinh HQ, Huang P, Lin L, Liu X, Ding LW, Wang J, Berman BP, Song EW, et al. Genomic and Epigenomic heterogeneity of hepatocellular carcinoma. Cancer Res. 2017;77(9):2255-65.

5. Nishida N, Kudo M. Immune checkpoint blockade for the treatment of human hepatocellular carcinoma. Hepatol Res. 2018;48(8):622-34.

6. Xiang DM, Sun W, Zhou T, Zhang C, Cheng Z, Li SC, Jiang W, Wang R, Fu G, Cui $X$, et al. Oncofetal HLF transactivates C-Jun to promote hepatocellular carcinoma development and sorafenib resistance. Gut. 2019.

7. Yin Z, Jiang K, Li R, Dong C, Wang L. Multipotent mesenchymal stromal cells play critical roles in hepatocellular carcinoma initiation, progression and therapy. Mol Cancer. 2018;17(1):178.

8. Ji J, Yin Y, Ju H, Xu X, Liu W, Fu Q, Hu J, Zhang X, Sun B. Long non-coding RNA Lnc-Tim3 exacerbates CD8 T cell exhaustion via binding to Tim-3 and inducing nuclear translocation of Bat3 in HCC. Cell Death Dis. 2018;9(5):478.

9. Wang $X$, He Q, Shen $H$, Xia A, Tian W, Yu W, Sun B. TOX promotes the exhaustion of antitumor CD8(+) T cells by preventing PD1 degradation in hepatocellular carcinoma. J Hepatol. 2019.

10. Ju Y, Hou N, Meng J, Wang X, Zhang X, Zhao D, Liu Y, Zhu F, Zhang L, Sun $W$, et al. T cell immunoglobulin- and mucin-domain-containing molecule-3 (Tim-3) mediates natural killer cell suppression in chronic hepatitis B. J Hepatol. 2010;52(3):322-9.

11. Sanchez-Correa B, Lopez-Sejas N, Duran E, Labella F, Alonso C, Solana R, Tarazona R. Modulation of NK cells with checkpoint inhibitors in the context of cancer immunotherapy. Cancer Immunol Immunother. 2019;68(5):861-70.

12. Lee H, Quek C, Silva I, Tasker A, Batten M, Rizos H, Lim SY, Nur Gide T, Shang P, Attrill GH, et al. Integrated molecular and immunophenotypic analysis of NK cells in anti-PD-1 treated metastatic melanoma patients. Oncoimmunology. 2019;8(2):e1537581.

13. Barry KC, Hsu J, Broz ML, Cueto FJ, Binnewies M, Combes AJ, Nelson AE, Loo K, Kumar R, Rosenblum MD, et al. A natural killer-dendritic cell axis defines checkpoint therapy-responsive tumor microenvironments. Nat Med. 2018; 24(8):1178-91.

14. Mudbhary R, Hoshida Y, Chernyavskaya Y, Jacob V, Villanueva A, Fiel MI, Chen X, Kojima K, Thung S, Bronson RT, et al. UHRF1 overexpression drives DNA hypomethylation and hepatocellular carcinoma. Cancer Cell. 2014; 25(2):196-209.

15. Tian XP, Wang CY, Jin XH, Li M, Wang FW, Huang WJ, Yun JP, Xu RH, Cai $\mathrm{QQ}$, Xie D. Acidic microenvironment up-regulates Exosomal miR-21 and miR-10b in early-stage hepatocellular carcinoma to promote Cancer cell proliferation and metastasis. Theranostics. 2019;9(7):1965-79.

16. Li L, Li C, Wang S, Wang Z, Jiang J, Wang W, Li X, Chen J, Liu K, Li C, et al. Exosomes derived from hypoxic Oral squamous cell carcinoma cells deliver miR-21 to normoxic cells to elicit a Prometastatic phenotype. Cancer Res. 2016;76(7):1770-80. 
17. Wei JX, Lv LH, Wan YL, Cao Y, Li GL, Lin HM, Zhou R, Shang CZ, Cao J, He H, et al. Vps4A functions as a tumor suppressor by regulating the secretion and uptake of exosomal microRNAs in human hepatoma cells. Hepatology. 2015;61(4):1284-94.

18. Zhang PF, Wei CY, Huang XY, Peng R, Yang X, Lu JC, Zhang C, Gao C, Cai JB, Gao PT, et al. Circular RNA circTRIM33-12 acts as the sponge of MicroRNA-191 to suppress hepatocellular carcinoma progression. Mol Cancer. 2019;18(1):105

19. Liu H, Bi J, Dong W, Yang M, Shi J, Jiang N, Lin T, Huang J. Invasion-related circular RNA circFNDC3B inhibits bladder cancer progression through the miR-1178-3p/G3BP2/SRC/FAK axis. Mol Cancer. 2018;17(1):161.

20. Dong W, Bi J, Liu H, Yan D, He Q, Zhou Q, Wang Q, Xie R, Su Y, Yang M, et al. Circular RNA ACVR2A suppresses bladder cancer cells proliferation and metastasis through miR-626/EYA4 axis. Mol Cancer. 2019;18(1):95.

21. Wei CY, Zhu MX, Yang YW, Zhang PF, Yang X, Peng R, Gao C, Lu JC, Wang $L$, Deng $X Y$, et al. Downregulation of RNF128 activates Wnt/beta-catenin signaling to induce cellular EMT and stemness via CD44 and CTTN ubiquitination in melanoma. J Hematol Oncol. 2019;12(1):21.

22. Chen LL. The biogenesis and emerging roles of circular RNAs. Nat Rev Mol Cell Biol. 2016;17(4):205-11.

23. Shang BQ, Li ML, Quan HY, Hou PF, Li ZW, Chu SF, Zheng JN, Bai J. Functional roles of circular RNAs during epithelial-to-mesenchymal transition. Mol Cancer. 2019;18(1):138.

24. Shan C, Zhang Y, Hao X, Gao J, Chen X, Wang K. Biogenesis, functions and clinical significance of circRNAs in gastric cancer. Mol Cancer. 2019;18(1):136.

25. Kristensen LS, Andersen MS, Stagsted LW, Ebbesen KK, Hansen TB, Kjems J. The biogenesis, biology and characterization of circular RNAs. Nat Rev Genet. 2019:20(11):675-91.

26. Zhou R, Chen KK, Zhang J, Xiao B, Huang Z, Ju C, Sun J, Zhang F, Lv XB, Huang G. The decade of exosomal long RNA species: an emerging cancer antagonist. Mol Cancer. 2018;17(1):75.

27. Zhang X, Wang S, Wang H, Cao J, Huang X, Chen Z, Xu P, Sun G, Xu J, Lv J, et al. Circular RNA circNRIP1 acts as a microRNA-149-5p sponge to promote gastric cancer progression via the AKT1/mTOR pathway. Mol Cancer. 2019; 18(1):20.

28. Zhang PF, Pei X, Li KS, Jin LN, Wang F, Wu J, Zhang XM. Circular RNA circFGFR1 promotes progression and anti-PD-1 resistance by sponging miR381-3p in non-small cell lung cancer cells. Mol Cancer. 2019;18(1):179.

29. Yu J, Xu QG, Wang ZG, Yang Y, Zhang L, Ma JZ, Sun SH, Yang F, Zhou WP. Circular RNA cSMARCA5 inhibits growth and metastasis in hepatocellular carcinoma. J Hepatol. 2018;68(6):1214-27.

30. Holdt LM, Stahringer A, Sass K, Pichler G, Kulak NA, Wilfert W, Kohlmaier A, Herbst A, Northoff BH, Nicolaou A, et al. Circular non-coding RNA ANRIL modulates ribosomal RNA maturation and atherosclerosis in humans. Nat Commun. 2016:7:12429.

31. Zheng Y, Li Y, Lian J, Yang H, Li F, Zhao S, Qi Y, Zhang Y, Huang L. TNFalpha-induced Tim-3 expression marks the dysfunction of infiltrating natural killer cells in human esophageal cancer. J Transl Med. 2019;17(1):165.

32. da Silva IP, Gallois A, Jimenez-Baranda S, Khan S, Anderson AC, Kuchroo VK, Osman I, Bhardwaj N. Reversal of NK-cell exhaustion in advanced melanoma by Tim-3 blockade. Cancer Immunol Res. 2014;2(5):410-22.

33. Tang R, Rangachari M, Kuchroo VK. Tim-3: a co-receptor with diverse roles in T cell exhaustion and tolerance. Semin Immunol. 2019;42:101302.

34. Chen X, Yang T, Wang W, Xi W, Zhang T, Li Q, Yang A, Wang T. Circular RNAs in immune responses and immune diseases. Theranostics. 2019;9(2): 588-607.

35. Ng WL, Mohd Mohidin TB, Shukla K. Functional role of circular RNAs in cancer development and progression. RNA Biol. 2018;15(8):995-1005.

36. Kristensen LS, Andersen MS, Stagsted LWW, Ebbesen KK, Hansen TB, Kjems J. The biogenesis, biology and characterization of circular RNAs. Nat Rev Genet. 2019.

37. Kim DH, Kim H, Choi YJ, Kim SY, Lee JE, Sung KJ, Sung YH, Pack CG, Jung MK, Han B, et al. Exosomal PD-L1 promotes tumor growth through immune escape in non-small cell lung cancer. Exp Mol Med. 2019;51(8):94.

38. Kulkarni B, Kirave P, Gondaliya P, Jash K, Jain A, Tekade RK, Kalia K. Exosomal miRNA in chemoresistance, immune evasion, metastasis and progression of cancer. Drug Discov Today. 2019

39. Wang Y, Liu J, Ma J, Sun T, Zhou Q, Wang W, Wang G, Wu P, Wang H, Jiang $L$, et al. Exosomal circRNAs: biogenesis, effect and application in human diseases. Mol Cancer. 2019:18(1):116.
40. Xie Y, Dang W, Zhang S, Yue W, Yang L, Zhai X, Yan Q, Lu J. The role of exosomal noncoding RNAs in cancer. Mol Cancer. 2019;18(1):37.

41. Wang G, Liu W, Zou Y, Wang G, Deng Y, Luo J, Zhang Y, Li H, Zhang Q, Yang $Y$, et al. Three isoforms of exosomal circPTGR1 promote hepatocellular carcinoma metastasis via the miR449a-MET pathway. EBioMedicine. 2019;40: 432-45.

42. Li S, Li Y, Chen B, Zhao J, Yu S, Tang Y, Zheng Q, Li Y, Wang P, He X, et al. exoRBase: a database of circRNA, IncRNA and mRNA in human blood exosomes. Nucleic Acids Res. 2018;46(D1):D106-12.

43. Chen EB, Zhou ZJ, Xiao K, Zhu GQ, Yang Y, Wang B, Zhou SL, Chen Q, Yin D, Wang $Z$, et al. The miR-561-5p/CX3CL1 signaling Axis regulates pulmonary metastasis in hepatocellular carcinoma involving CX3CR1(+) natural killer cells infiltration. Theranostics. 2019;9(16):4779-94.

44. Terry S, Abdou A, Engelsen AST, Buart S, Dessen P, Corgnac S, Collares D, Meurice G, Gausdal G, Baud V, et al. AXL targeting overcomes human lung cancer cell resistance to NK and CTL-mediated cytotoxicity. Cancer Immuno Res. 2019.

45. Lopez-Soto A, Gonzalez S, Smyth MJ, Galluzzi L. Control of metastasis by NK cells. Cancer Cell. 2017;32(2):135-54

46. Marin-Acevedo JA, Dholaria B, Soyano AE, Knutson KL, Chumsri S, Lou Y. Next generation of immune checkpoint therapy in cancer: new developments and challenges. J Hematol Oncol. 2018:11(1):39.

47. Waidmann O. Recent developments with immunotherapy for hepatocellular carcinoma. Expert Opin Biol Ther. 2018;18(8):905-10.

48. Sun H, Liu L, Huang Q, Liu H, Huang M, Wang J, Wen H, Lin R, Qu K, Li K, et al. Accumulation of tumor-infiltrating CD49a(+) NK cells correlates with poor prognosis for human hepatocellular carcinoma. Cancer Immunol Res. 2019;7(9):1535-46.

49. Trefny MP, Rothschild SI, Uhlenbrock F, Rieder D, Kasenda B, Stanczak MA, Berner F, Kashyap AS, Kaiser M, Herzig P, et al. A variant of a killer cel immunoglobulin-like receptor is associated with resistance to PD-1 blockade in lung Cancer. Clin Cancer Res. 2019;25(10):3026-34.

50. Zhang Q, Bi J, Zheng X, Chen Y, Wang H, Wu W, Wang Z, Wu Q, Peng H, Wei $\mathrm{H}$, et al. Blockade of the checkpoint receptor TIGIT prevents NK cell exhaustion and elicits potent anti-tumor immunity. Nat Immunol. 2018; 19(7):723-32.

51. Gallois A, Silva I, Osman I, Bhardwaj N. Reversal of natural killer cell exhaustion by TIM-3 blockade. Oncoimmunology. 2014;3(12):e946365

52. Nayyar G, Chu Y, Cairo MS. Overcoming resistance to natural killer cell based immunotherapies for solid tumors. Front Oncol. 2019;9:51.

53. Guillerey C, Huntington ND, Smyth MJ. Targeting natural killer cells in cancer immunotherapy. Nat Immunol. 2016;17(9):1025-36.

\section{Publisher's Note}

Springer Nature remains neutral with regard to jurisdictional claims in published maps and institutional affiliations.
Ready to submit your research? Choose BMC and benefit from:

- fast, convenient online submission

- thorough peer review by experienced researchers in your field

- rapid publication on acceptance

- support for research data, including large and complex data types

- gold Open Access which fosters wider collaboration and increased citations

- maximum visibility for your research: over $100 \mathrm{M}$ website views per year

At $\mathrm{BMC}$, research is always in progress.

Learn more biomedcentral.com/submission 